\title{
SPATIALLY RESOLVED X-RAY SPECTRA OF CORONAL ACTIVE REGIONS
}

\author{
R. C. CATURA, L. W. ACTON, and E. G. JOKI \\ Lockheed Palo Alto Laboratories, U.S.A. \\ and \\ C. G. RA PLEY and J. L. CULHANE \\ Mullard Space Science Laboratory, University College London, Engiand
}

Summary. X-ray spectra from a number of coronal active regions were obtained during ATM support rocket flights carried out by the Lockheed group on June 11 and December 19, 1973. Multi-grid collimators were used to provide fields of view of $40^{\prime \prime}$ diameter and $90^{\prime \prime}$ diameter for a number of scanning crystal spectrometers and a bent crystal spectrometer which employed a position sensitive proportional counter to register the diffracted spectrum. A solar image was produced on film and on a TV camera on board the rocket with the aid of a $1 \AA \mathrm{H} \alpha$ filter. A small part of the X-ray collimator was used to generate a multiple spot diffraction pattern which was superimposed on the $\mathrm{H} \alpha$ image and the composite picture was transmitted to the ground. Pre-launch calibrations allowed the spot corresponding to the X-ray collimator axis to be identified and so the collimator pointing direction on the solar disc was controlled from the ground by means of commands sent to the rocket.

In the June 11 flight, spectra of both active region material and of the quiet corona were obtained in a number of different wavelength ranges with the scanning spectrometers and included emission features of $\mathrm{Ne}$ IX, Fe XVII, $\mathrm{O}$ VIII and $\mathrm{O}$ VII. The bent crystal spectrometer obtained a spectrum in the range 10-18 $\AA$ and so established the usefullness of this new technique (Catura et al., 1974). For the flight of December 19, all of the spectrometers were collimated to $40^{\prime \prime}$. The six wavelength ranges scanned by the plane spectrometers included emission features of $\mathrm{Mg} \mathrm{XI}, \mathrm{Ne} I x, \mathrm{Ne} \mathrm{x}, \mathrm{Fe}$ XVII, Fe XVIII, $O$ VIII and $O$ VII. The bent crystal spectrometer was used in the range 16-17.2 $\AA$ and demonstrated its ability to resolve the closely spaced $3 p_{1}$ and $3 p_{2}$ Fe XVII lines at $17.041 \AA$ and $17.086 \AA$.

Comparison of the collimated spectra with X-ray images obtained with the S0-56 telescope on ATM, kindly supplied by Dr J. H. Underwood, established that the higher temperature material is concentrated in compact features surrounded by more extended cooler material. The corona outside of the active regions was only detected in the lines of $\mathrm{O}$ VII and $\mathrm{O}$ vIII. Further analysis of these data will include a search for density effects in the He-like line ratios, the estimation of temperature from satellite line intensities and the construction of models of the relation between emission measure and temperature for different parts of the active regions.

The Lockheed work was supported by NASA-Ames Research Center.

\section{References}

Catura, R. C., Joki, E. G., Bakka, J. C., Rapley, C. G., and Culhane, J. L.: 1974, Monthly Notices Roy. Astron. Soc. 168, 217. 